\title{
Comparative study on optimizing closed-loop stability bounds of finite-precision controller structures with shift and delta operators
}

\author{
S. Chen ${ }^{\mathrm{a}, *}$, R.H. Istepanian ${ }^{\mathrm{b}}, \mathrm{J} \cdot \mathrm{Wu}^{\mathrm{c}}, \mathrm{J} \cdot \mathrm{Chu}^{\mathrm{c}}$ \\ ${ }^{a}$ Department of Electronics and Computer Science, University of Southampton, Highfield, Southampton SO17 1BJ, UK \\ ${ }^{\mathrm{b}}$ Department of Electrical and Computer Engineering, Ryerson Polytechnic University, 350 Victoria Street, Toronto, Ontario, \\ Canada M5B $2 K 3$ \\ ${ }^{\mathrm{c}}$ National Laboratory of Industrial Control Technology, Institute of Industrial Process Control, Zhejiang University, Hangzhou, \\ 310027, People's Republic of China
}

\begin{abstract}
The paper analyzes the sensitivity of closed-loop stability with respect to (w.r.t.) finite word length (FWL) effects in the implementation of the controller coefficients using both the shift and delta operator parameterizations. A unified approach is established to optimize a closed-loop stability lower bound for FWL controller structures. This provides a general framework to compare the FWL closed-loop stability characteristics of the controller structures based on the shift and delta operators, respectively. Two numerical examples are given, and the simulation results show that the optimal FWL controller realizations with the delta operator have better closed-loop stability margins than those with the shift operator. (c) 2000 Elsevier Science B.V. All rights reserved.
\end{abstract}

Keywords: Sampled-data system; Digital controller; Finite word length; Stability; Shift and delta operators; Optimization

\section{Introduction}

Controller implementations with fixed-point arithmetic offer the advantages of speed, memory space and simplicity over floating-point arithmetic. However, a designed stable closed-loop system may become unstable when the infinite-precision controller is implemented using a fixed-point processor due to FWL effects. The "robustness" of closed-loop stability w.r.t. controller parameter perturbations therefore is a critical issue in fixed-point implementations of digital controllers. A discrete-time system can be described and realized either with the usual shift $z$ operator or the delta $\delta$ operator [12]. Two major advantages are claimed for the use of $\delta$ operator realization: a theoretically unified formulation of continuous-time and discrete-time systems; and better numerical properties in FWL implementations [8].

The issues of FWL controller realizations in the $z$ domain have been addressed [7,11,10]. Investigations on finite-precision controller realizations with the $z$ operator parameterization and relevant optimization problems

\footnotetext{
* Corresponding author. Tel./Fax: +44-(0)23-8059-6660/4508.

E-mail address: sqc@ecs.soton.ac.uk (S. Chen).
} 
have mainly been based on the sensitivity of the closed-loop pole positions w.r.t. FWL effects $[11,10,3]$. This approach has recently been extended to study the closed-loop stability issues of FWL controller structures using the $\delta$ operator formulation [14,4]. In this paper, we provide a comparative study on optimizing closed-loop stability bounds of finite-precision controller structures with the $z$ and $\delta$ operator parameterizations.

We present a unified approach for analyzing the sensitivity of closed-loop stability w.r.t. FWL effects for both the $z$ and $\delta$ parameterizations. A tractable lower-bound stability measure is provided. The optimal FWL controller realization, which maximizes this lower-bound stability measure, can be obtained by solving a constrained optimization problem. For the PID controller, this constrained optimization can be decoupled into two unconstrained ones. As the optimization criteria are non-smooth and non-convex, a conventional optimization algorithm [1,6] may become trapped at some local minima and give misleading results. It is therefore critical to employ a global optimization method, such as genetic algorithms or adaptive simulated annealing (ASA) [9,2], in our comparative study. We adopt the ASA because of its simplicity in programming.

Two numerical examples, an IFAC benchmark PID control system [13] and a control system with a high-order controller [5], are used in the comparative study. The simulation results demonstrate that the $\delta$-based controller realization has better stability robustness to FWL effects than the $z$-domain approach, particularly for fast sampling. When the sampling rate increases, the closed-loop stability of the $z$-based FWL controller deteriorates significantly while the $\delta$-based FWL controller is actually improved slightly.

\section{Closed-loop stability with FWL controller structures}

We first define a "generalized" operator $\rho$ for discrete-time systems. It is understood that $\rho=z$ or $\delta$, depending on which operator is actually used. The $\delta$ operator is given by [12]

$$
\delta=\frac{z-1}{h},
$$

where $h$ is the sampling period. Given a transfer function $G(\rho)$, the state-space model is

$$
\begin{aligned}
& \rho x(k)=A_{\rho, G} x(k)+B_{\rho, G} u(k), \\
& y(k)=C_{\rho, G} x(k)+D_{\rho, G} u(k) .
\end{aligned}
$$

The state-space realization of an input-output transfer function is not unique. Define

$$
S_{\rho} \triangleq\left\{\left(A_{\rho, G}, B_{\rho, G}, C_{\rho, G}, D_{\rho, G}\right): G(\rho)=C_{\rho, G}\left(\rho I-A_{\rho, G}\right)^{-1} B_{\rho, G}+D_{\rho, G}\right\} .
$$

Then if $\left(A_{\rho, G}, B_{\rho, G}, C_{\rho, G}, D_{\rho, G}\right) \in S_{\rho},\left(\mathscr{T}^{-1} A_{\rho, G} \mathscr{T}, \mathscr{T}^{-1} B_{\rho, G}, C_{\rho, G} \mathscr{T}, D_{\rho, G}\right) \in S_{\rho}$ for any non-singular $\mathscr{T}$. The relationships relate the two state-space representations in the $z$ and $\delta$ operators are

$$
A_{z, G}=h A_{\delta, G}+I, \quad B_{z, G}=h B_{\delta, G}, \quad C_{z, G}=C_{\delta, G}, \quad D_{z, G}=D_{\delta, G} .
$$

Let $\left\{\lambda_{\rho, i}\right\}$ be the eigenvalues of $A_{\rho, G}$. The following lemma relates $\left\{\lambda_{\delta, i}\right\}$ to $\left\{\lambda_{z, i}\right\}$.

Lemma 1. $\lambda_{z, i}=1+h \lambda_{\delta, i}, \forall i$.

It is well known that the discrete-time system $\left(A_{z, G}, B_{z, G}, C_{z, G}, D_{z, G}\right)$ is stable if and only if all the eigenvalues $\left|\lambda_{z, i}\right|<1$. From Lemma 1 , we have the condition of the stability of the discrete-time system described with the $\delta$ operator.

Lemma 2. The discrete-time system $\left(A_{\delta, G}, B_{\delta, G}, C_{\delta, G}, D_{\delta, G}\right)$ is stable if and only if

$$
\left|\lambda_{\delta, i}+\frac{1}{h}\right|<\frac{1}{h}, \quad \forall i .
$$

Consider the sampled-data system depicted in Fig. 1, where the continuous-time plant $P(s)$ is strictly proper. 


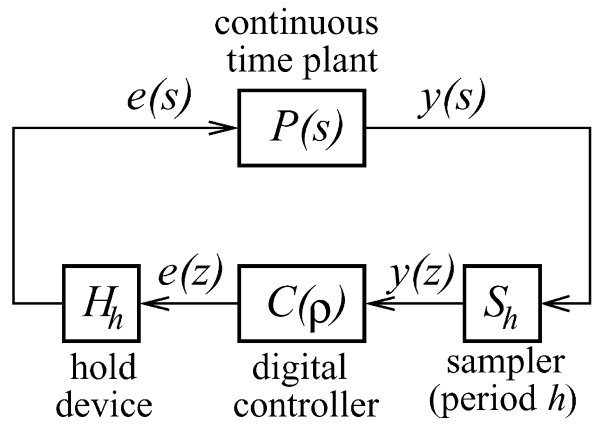

Fig. 1. Sampled-data system with digital controller realization, where $\rho=z$ or $\delta$.

The discrete-time plant $P(\rho)=S_{h} P(s) H_{h}$ has a state-space realization $\left(A_{\rho, P} \in \mathbb{R}^{m \times m}, B_{\rho, P} \in \mathbb{R}^{m \times l}, C_{\rho, P} \in\right.$ $\left.\mathbb{R}^{q \times m}, 0\right)$. The controller $C(\rho)$ has a state-space realization $\left(A_{\rho, C} \in \mathbb{R}^{n \times n}, B_{\rho, C} \in \mathbb{R}^{n \times q}, C_{\rho, C} \in \mathbb{R}^{l \times n}, D_{\rho, C} \in\right.$ $\left.\mathbb{R}^{l \times q}\right)$. The corresponding state-space realization $\left(\bar{A}_{\rho}, \bar{B}_{\rho}, \bar{C}_{\rho}, \bar{D}_{\rho}\right)$ of the closed-loop system is

$$
\begin{aligned}
\bar{A}_{\rho} & =\left[\begin{array}{cc}
A_{\rho, P} & 0 \\
0 & 0
\end{array}\right]+\left[\begin{array}{cc}
B_{\rho, P} & 0 \\
0 & I_{n}
\end{array}\right]\left[\begin{array}{ll}
D_{\rho, C} & C_{\rho, C} \\
B_{\rho, C} & A_{\rho, C}
\end{array}\right]\left[\begin{array}{cc}
C_{\rho, P} & 0 \\
0 & I_{n}
\end{array}\right] \\
& =M_{0}+M_{1} X_{\rho} M_{2}=\bar{A}_{\rho}\left(X_{\rho}\right), \\
\bar{B}_{\rho} & =\left[\begin{array}{c}
B_{\rho, P} \\
0
\end{array}\right], \quad \bar{C}_{\rho}=\left[C_{\rho, P} 0\right], \quad \bar{D}_{\rho}=0,
\end{aligned}
$$

where

$$
X_{\rho}=\left[\begin{array}{cc}
D_{\rho, C} & C_{\rho, C} \\
B_{\rho, C} & A_{\rho, C}
\end{array}\right]=\left[\begin{array}{cccc}
p_{1} & p_{2} & \cdots & p_{q+n} \\
p_{q+n+1} & p_{q+n+2} & \cdots & p_{2(q+n)} \\
\vdots & \vdots & \cdots & \vdots \\
p_{(l+n-1)(q+n)+1} & p_{(l+n-1)(q+n)+2} & \cdots & p_{(l+n)(q+n)}
\end{array}\right]
$$

is the controller matrix. Denote the eigenvalues of $\bar{A}_{\rho}\left(X_{\rho}\right)$ by $\left\{\bar{\lambda}_{\rho, i}, 1 \leqslant i \leqslant m+n\right\}$. We define the "stability margin" of $\bar{\lambda}_{\rho, i}$ as

$$
\operatorname{StMa}\left(\bar{\lambda}_{\rho, i}\right)= \begin{cases}1-\left|\bar{\lambda}_{z, i}\right|, & \rho=z, \\ \frac{1}{h}-\left|\bar{\lambda}_{\delta, i}+\frac{1}{h}\right|, & \rho=\delta .\end{cases}
$$

Let $C(\rho)$ be chosen to make the closed-loop system stable. Then $\operatorname{StMa}\left(\bar{\lambda}_{\rho, i}\right)>0, \forall i$.

When the realization $\left(A_{\rho, C}, B_{\rho, C}, C_{\rho, C}, D_{\rho, C}\right)$ is implemented with a fixed-point processor, $X_{\rho}$ is perturbed into $X_{\rho}+\Delta X_{\rho}$ due to the FWL effects, where

$$
\Delta X_{\rho}=\left[\begin{array}{cccc}
\Delta p_{1} & \Delta p_{2} & \cdots & \Delta p_{q+n} \\
\Delta p_{q+n+1} & \Delta p_{q+n+2} & \cdots & \Delta p_{2(q+n)} \\
\vdots & \vdots & \cdots & \vdots \\
\Delta p_{(l+n-1)(q+n)+1} & \Delta p_{(l+n-1)(q+n)+2} & \cdots & \Delta p_{N}
\end{array}\right]
$$

and $N=(l+n)(q+n)$. Each element of $\Delta X_{\rho}$ is bounded by $\varepsilon / 2$, that is,

$$
\mu\left(\Delta X_{\rho}\right) \triangleq \max _{1 \leqslant i \leqslant N}\left|\Delta p_{i}\right| \leqslant \frac{\varepsilon}{2} .
$$

For a fixed-point processor of $B_{\mathrm{s}}$ bits, $\varepsilon=2^{-\left(B_{\mathrm{s}}-B_{X}\right)}$, and $2^{B_{X}}$ is a normalization factor such that the absolute value of each element of $2^{-B_{X}} X_{\rho}$ is not larger than 1 . With the perturbation $\Delta X_{\rho}, \bar{\lambda}_{\rho, i}$ is moved to $\tilde{\lambda}_{\rho, i}$. The 
sampled-data system will be unstable if there exists $i \in\{1, \ldots, m+n\}$ such that $\operatorname{StMa}\left(\tilde{\lambda}_{\rho, i}\right) \leqslant 0$. To see when the round off error will cause the closed-loop system to become unstable, define the stability measure

$$
\mu_{\rho, 0}\left(X_{\rho}\right) \triangleq \inf \left\{\mu\left(\Delta X_{\rho}\right): \bar{A}_{\rho}\left(X_{\rho}+\Delta X_{\rho}\right) \text { is unstable }\right\} .
$$

From this definition, it is obvious that:

Proposition 1. $\bar{A}_{\rho}\left(X_{\rho}+\Delta X_{\rho}\right)$ is stable if $\mu\left(\Delta X_{\rho}\right)<\mu_{\rho, 0}\left(X_{\rho}\right)$.

The large $\mu_{\rho, 0}\left(X_{\rho}\right)$ is, the bigger FWL error the closed-loop can tolerate. For a given $X_{\rho}$, the closed-loop stability is guaranteed if the FWL error lies in the bounded region:

$$
\mathscr{Z}\left(X_{\rho}\right) \triangleq\left\{\Delta X_{\rho}: \mu\left(\Delta X_{\rho}\right)<\mu_{\rho, 0}\left(X_{\rho}\right)\right\} .
$$

Thus, $\mu_{\rho, 0}\left(X_{\rho}\right)$ quantifies the stability robustness of the realization $X_{\rho}$ to the FWL effects. However, computing explicitly the value of $\mu_{\rho, 0}\left(X_{\rho}\right)$ is an unsolved open problem. For the computational purpose, we define the following tractable alternative:

$$
\mu_{\rho, 1}\left(X_{\rho}\right)=\min _{1 \leqslant i \leqslant m+n} \frac{\operatorname{StMa}\left(\bar{\lambda}_{\rho, i}\right)}{\sum_{j=1}^{N}\left|\partial \bar{\lambda}_{\rho, i} / \partial p_{j}\right|_{X_{\rho}} \mid} .
$$

Proposition 2. There exists a rather large $\kappa>0$ such that

$$
\forall \Delta X_{\rho} \in \mathscr{P}\left(X_{\rho}\right) \triangleq\left\{\Delta X_{\rho}: \mu\left(\Delta X_{\rho}\right) \leqslant \kappa\right\},
$$

$\bar{A}_{\rho}\left(X_{\rho}+\Delta X_{\rho}\right)$ is stable if $\mu\left(\Delta X_{\rho}\right)<\mu_{\rho, 1}\left(X_{\rho}\right)$.

The proof of this proposition for the case of $\rho=\delta$ is given in [4]. The requirement of $\Delta X_{\rho} \in \mathscr{P}\left(X_{\rho}\right)$ is not over restricted, since $\mathscr{P}\left(X_{\rho}\right)$ covers at least a large part of $\mathscr{2}\left(X_{\rho}\right)$.

Corollary 1. $\mu_{\rho, 1}\left(X_{\rho}\right) \leqslant \mu_{\rho, 0}\left(X_{\rho}\right)$ if

$$
\mu_{\rho, 0}\left(X_{\rho}\right)<\inf _{\Delta X_{\rho} \notin \mathscr{P}\left(X_{\rho}\right)} \mu\left(\Delta X_{\rho}\right) .
$$

Thus, $\mu_{\rho, 1}\left(X_{\rho}\right)$ can be considered as a lower bound of $\mu_{\rho, 0}\left(X_{\rho}\right)$, provided that $\mu_{\rho, 0}\left(X_{\rho}\right)$ is small enough. The following theorem shows that $\mu_{\rho, 1}\left(X_{\rho}\right)$ can be computed easily.

Theorem 1. Let $\left\{\bar{\lambda}_{\rho, i}, 1 \leqslant i \leqslant m+n\right\}$ be the eigenvalues of $\bar{A}_{\rho}\left(X_{\rho}\right)$, and $\boldsymbol{x}_{i}$ and $\boldsymbol{y}_{i}$ be the right and reciprocal left eigenvectors corresponding to $\bar{\lambda}_{\rho, i}$, respectively. Then

$$
\frac{\partial \bar{\lambda}_{\rho, i}}{\partial X_{\rho}}=\left[\begin{array}{cccc}
\frac{\partial \bar{\lambda}_{\rho, i}}{\partial p_{1}} & \frac{\partial \bar{\lambda}_{\rho, i}}{\partial p_{2}} & \cdots & \frac{\partial \bar{\lambda}_{\rho, i}}{\partial p_{q+n}} \\
\frac{\partial \bar{\lambda}_{\rho, i}}{\partial p_{q+n+1}} & \frac{\partial \bar{\lambda}_{\rho, i}}{\partial p_{q+n+2}} & \cdots & \frac{\partial \bar{\lambda}_{\rho, i}}{\partial p_{2(q+n)}} \\
\vdots & \vdots & \cdots & \vdots \\
\frac{\partial \bar{\lambda}_{\rho, i}}{\partial p_{(l+n-1)(q+n)+1}} & \frac{\partial \bar{\lambda}_{\rho, i}}{\partial p_{(l+n-1)(q+n)+2}} & \cdots & \frac{\partial \bar{\lambda}_{\rho, i}}{\partial p_{N}}
\end{array}\right]=M_{1}^{\mathrm{T}} \boldsymbol{y}_{i}^{*} \boldsymbol{x}_{i}^{\mathrm{T}} M_{2}^{\mathrm{T}},
$$

where $\mathrm{T}$ denotes the transpose operation, and ${ }^{*}$ the conjugate operation.

The proof of this theorem is identical to the special case of $\rho=z$ given in $[11,10]$.

How "robust" a controller is to the FWL effects can also be viewed from another angle. Let $B_{\mathrm{s}}^{\min }$ be the smallest word length that can guarantee the closed-loop stability. It would be highly desirable to know $B_{\mathrm{s}}^{\mathrm{min}}$ 
for a given realization $X_{\rho}$. However, except in simulation, it is impossible to test the closed-loop system by reducing $B_{\mathrm{s}}$ until it becomes unstable. An estimate of $B_{\mathrm{s}}^{\min }$ is given by

$$
\hat{B}_{\mathrm{s}}^{\min }=\operatorname{Int}\left[-\log _{2}\left(\mu_{\rho, 1}\left(X_{\rho}\right)\right)\right]-1+B_{X},
$$

where $\operatorname{Int}[x]$ rounds $x$ to the nearest integer and $\operatorname{Int}[x] \geqslant x$. From (11) and Proposition 2, it can be seen that the closed-loop system is stable when $X_{\rho}$ is implemented with a fixed-point processor of at least $\hat{B}_{\mathrm{s}}^{\mathrm{min}}$ bits.

It is worth emphasizing a practical consideration on the FWL implementation of $\delta$-based controllers. The state-space equation of the $\delta$-based controller

$$
\delta x(k)=A_{\delta, C} x(k)+B_{\delta, C} u(k)
$$

is realized using

$$
x(k+1)=x(k)+h\left(A_{\delta, C} x(k)+B_{\delta, C} u(k)\right) .
$$

The sampling period $h$ should be implemented exactly without any FWL errors. Otherwise, analysis based on $X_{\delta}$ may not be valid. Assume that $h$ can be realized exactly by $B_{h}$ bits with the integer part of $h$ requiring $B_{h I}$ bits and the fractional part of $h$ requiring $B_{h F}$ bits. A modified estimate of $B_{\mathrm{s}}^{\min }$ is

$$
\hat{B}_{s h}^{\min }=\max \left\{B_{h I}, B_{X}\right\}+\max \left\{B_{h F}, \hat{B}_{\mathrm{s}}^{\min }-B_{X}\right\} .
$$

This modified estimate can be pessimistic, but the estimate (17) is not always correct for $\delta$ realizations. Controllers based on the $z$ operator do not have this problem.

\section{Optimal FWL controller realizations}

From the previous discussions, we know that there are different realizations $X_{\rho}$ for a given $C(\rho)$, and the stability measure $\mu_{\rho, 1}\left(X_{\rho}\right)$ is a function of the realization. It is of practical importance to find a realization such that $\mu_{\rho, 1}\left(X_{\rho}\right)$ is maximized. Such a realization is optimal in the sense that it has maximum stability robustness to FWL effects. The digital controller implemented with an optimal realization means that the stability of the closed-loop system is guaranteed with a minimum hardware requirement in terms of word length. Given an initial realization $X_{\rho, 0}$ of $C(\rho)$,

$$
X_{\rho, 0}=\left[\begin{array}{cc}
D_{\rho, C}^{0} & C_{\rho, C}^{0} \\
B_{\rho, C}^{0} & A_{\rho, C}^{0}
\end{array}\right],
$$

any realization of $C(\rho)$ can be expressed as

$$
X_{\rho, \mathscr{T}} \triangleq\left[\begin{array}{cc}
I_{l} & 0 \\
0 & \mathscr{T}^{-1}
\end{array}\right] X_{\rho, 0}\left[\begin{array}{cc}
I_{q} & 0 \\
0 & \mathscr{T}
\end{array}\right],
$$

where $\mathscr{T} \in \mathbb{R}^{n \times n}$ and $\operatorname{det}(\mathscr{T}) \neq 0$. From (6), the transition matrix of the closed-loop system is

$$
\bar{A}_{\rho}\left(X_{\rho, \mathscr{T}}\right)=\left[\begin{array}{cc}
I_{m} & 0 \\
0 & \mathscr{T}^{-1}
\end{array}\right] \bar{A}_{\rho}\left(X_{\rho, 0}\right)\left[\begin{array}{cc}
I_{m} & 0 \\
0 & \mathscr{T}
\end{array}\right] .
$$

Since the eigenvalues of $\bar{A}_{\rho}\left(X_{\rho, 0}\right)$ and $\bar{A}_{\rho}\left(X_{\rho, \mathscr{T}}\right)$ are identical, the same symbol $\bar{\lambda}_{\rho, i}$ is used to denote their $i$-th eigenvalue. From (23), applying Theorem 1 results in

$$
\left.\frac{\partial \bar{\lambda}_{\rho, i}}{\partial X}\right|_{X=X_{\rho, \mathscr{T}}}=\left.\left[\begin{array}{cc}
I_{l} & 0 \\
0 & \mathscr{T}^{T}
\end{array}\right] \frac{\partial \bar{\lambda}_{\rho, i}}{\partial X}\right|_{X=X_{\rho, 0}}\left[\begin{array}{cc}
I_{q} & 0 \\
0 & \mathscr{T}^{-T}
\end{array}\right] .
$$

From (14), (16) and (24), we can define the optimal controller realization as the solution of the following maximization problem:

$$
\varphi_{\rho} \triangleq \max _{\substack{\mathscr{T} \in \mathbb{R}^{n \times n} \\ \operatorname{det}(\mathscr{T}) \neq 0}} \mu_{\rho, 1}\left(X_{\rho, \mathscr{T}}\right)=\max _{\substack{\mathscr{T} \in \mathbb{R}^{n \times n} \\ \operatorname{det}(\mathscr{T}) \neq 0}} \min _{1 \leqslant i \leqslant m+n} \frac{\operatorname{StMa}\left(\bar{\lambda}_{\rho, i}\right)}{\sum_{j=1}^{N}\left|\partial \bar{\lambda}_{\rho, i} / \partial p_{j}\right|_{X=X_{\rho, \mathscr{T}}} \mid} .
$$


For the complex-valued matrix $M \in \mathbb{C}^{m \times n}$ with elements $M_{i, j}$, define a norm

$$
\|M\|_{\mathrm{s}} \triangleq \sum_{i=1}^{m} \sum_{j=1}^{n}\left|M_{i, j}\right|
$$

The optimization problem (25) is equivalent to the minimization problem

$$
v_{\rho}=\frac{1}{\varphi_{\rho}} \triangleq \min _{\substack{\mathscr{T} \in \mathbb{R}^{n \times n} \\ \operatorname{det}(\mathscr{T}) \neq 0}} f_{\rho}(\mathscr{T})
$$

with the cost function

$$
f_{\rho}(\mathscr{T})=\max _{1 \leqslant i \leqslant m+n}\left\|\left[\begin{array}{cc}
I_{l} & 0 \\
0 & \mathscr{T}^{T}
\end{array}\right] \Phi_{\rho, i}\left[\begin{array}{cc}
I_{q} & 0 \\
0 & \mathscr{T}^{-T}
\end{array}\right]\right\|_{S}
$$

and the constraint $\operatorname{det}(\mathscr{T}) \neq 0$, where

$$
\Phi_{\rho, i}=\frac{\partial \bar{\lambda}_{\rho, i} /\left.\partial X\right|_{X=X_{\rho, 0}}}{\operatorname{StMa}\left(\bar{\lambda}_{\rho, i}\right)}
$$

In practice, the constrained optimization (27) is solved by solving for the unconstrained optimization problem

$$
\tilde{v}_{\rho} \triangleq \min _{\mathscr{T} \in \mathbb{R}^{n \times n}} f_{\rho}(\mathscr{T})
$$

with a measure of monitoring the singular values of $\mathscr{T}$ to make sure that $\operatorname{det}(\mathscr{T}) \neq 0$. Because the cost function $f_{\rho}(\mathscr{T})$ is non-smooth and non-convex, non-gradient based optimization methods are required. The conventional non-gradient optimization algorithms, such as Rosenbrock and Simplex algorithms [1,6], in general can only obtain a local minimum solution. To overcome this difficulty, we adopt an efficient global optimization strategy based on the ASA. The ASA algorithm is detailed in [9,2].

\section{Optimal FWL PID controller realizations}

In this section, we specifically discuss the optimal realization problem of digital PID controllers. Let $\left(A_{\rho, C}^{0} \in\right.$ $\left.\mathbb{R}^{2 \times 2}, B_{\rho, C}^{0} \in \mathbb{R}^{2 \times 1}, C_{\rho, C}^{0} \in \mathbb{R}^{1 \times 2}, D_{\rho, C}^{0} \in \mathbb{R}\right)$ be an initial realization of the PID controller $C(\rho)$. From (27), the optimal PID controller realization problem is defined as

$$
v_{\rho} \triangleq \min _{\substack{\mathscr{T} \in \mathbb{R}^{2 \times 2} \\
\operatorname{det}(\mathscr{T}) \neq 0}} \max _{1 \leqslant i \leqslant m+2}\left\|\left[\begin{array}{cc}
1 & 0 \\
0 & \mathscr{T}^{T}
\end{array}\right] \Phi_{\rho, i}\left[\begin{array}{cc}
1 & 0 \\
0 & \mathscr{T}^{-T}
\end{array}\right]\right\|_{S} .
$$

The following theorem shows that the constrained optimization problem (31) can be decoupled into the two "simpler" unconstrained problems. First we define the two cost functions

$$
f_{\rho, 1}(x, y, w)=\max _{1 \leqslant i \leqslant m+2}\left\|\left[\begin{array}{ccc}
w & 0 & 0 \\
0 & x & 0 \\
0 & y & 1 / x
\end{array}\right] \Phi_{\rho, i}\left[\begin{array}{ccc}
1 / w & 0 & 0 \\
0 & 1 / x & 0 \\
0 & -y & x
\end{array}\right]\right\|_{S}
$$

and

$$
f_{\rho, 2}(x, y, u, w)=\max _{1 \leqslant i \leqslant m+2}\left\|\left[\begin{array}{ccc}
w & 0 & 0 \\
0 & x & u \\
0 & (x y-1) / u & y
\end{array}\right] \Phi_{\rho, i}\left[\begin{array}{ccc}
1 / w & 0 & 0 \\
0 & y & -u \\
0 & (1-x y) / u & x
\end{array}\right]\right\|_{s} .
$$

Theorem 2. Let

$$
v_{\rho, 1}=\min _{\substack{x \in(0,+\infty) \\ y \in(-\infty,+\infty) \\ w \in(0,+\infty)}} f_{\rho, 1}(x, y, w)
$$


and

$$
v_{\rho, 2}=\min _{\substack{x \in(-\infty,+\infty) \\ y \in(-\infty,+\infty) \\ u \in(0,+\infty) \\ w \in(0,+\infty)}} f_{\rho, 2}(x, y, u, w)
$$

Then

$$
v_{\rho}=\min \left\{v_{\rho, 1}, v_{\rho, 2}\right\} .
$$

If $v_{\rho}=v_{\rho, 1}$ and $\left(x_{\mathrm{opt} 1}, y_{\mathrm{opt} 1}, w_{\mathrm{opt} 1}\right)$ is the optimal solution of the problem (34), the optimal solution of the problem (31) is given as

$$
\mathscr{T}_{\mathrm{opt}}=\frac{1}{w_{\mathrm{opt} 1}}\left[\begin{array}{cc}
x_{\mathrm{opt} 1} & y_{\mathrm{opt} 1} \\
0 & 1 / x_{\mathrm{opt} 1}
\end{array}\right] .
$$

If $v_{\rho}=v_{\rho, 2}$ and $\left(x_{\mathrm{opt} 2}, y_{\mathrm{opt} 2}, u_{\mathrm{opt} 2}, w_{\mathrm{opt} 2}\right)$ is the optimal solution of the problem (35), the optimal solution of the problem (31) is given as

$$
\mathscr{T}_{\mathrm{opt}}=\frac{1}{w_{\mathrm{opt} 2}}\left[\begin{array}{lc}
x_{\mathrm{opt} 2} & \left(x_{\mathrm{opt} 2} y_{\mathrm{opt} 2}-1\right) / u_{\mathrm{opt} 2} \\
u_{\mathrm{opt} 2} & y_{\mathrm{opt} 2}
\end{array}\right] .
$$

The proof of Theorem 2 is identical to the special case of $\rho=z$ given in [3]. Because $f_{\rho, 1}(x, y, w)$ and $f_{\rho, 2}(x, y, u, w)$ are both non-smooth and non-convex functions, we will adopt the ASA algorithm to solve for the two corresponding optimization problems.

\section{Numerical examples}

Example 1. This was the IFAC93 benchmark PID control system [13]. The continuous-time plant model was

$$
P(s)=\frac{25(-0.4 s+1)}{\left(s^{2}+3 s+25\right)(5 s+1)}
$$

and the designed PID controller was

$$
C(s)=1.311+\frac{0.431}{s}+\frac{1.048 s}{1+12.92 s} .
$$

The sampled-data system with the infinite-precision controller was stable when the sampling period $h \leqslant 2^{3}$. The range of the sampling period used in the simulation was $2^{3}$ to $2^{-12}$. Given a sampling rate, the discrete-time plant model $P(\rho)$ and the digital controller $C(\rho)$ were obtained using the MATLAB discretizing routines, which are based on the bilinear (Tustin) transformation. The initial controller realization $X_{\rho, 0}$ was chosen to be the "controllable canonical" form. When $X_{\rho, 0}$ was provided, the eigenvalues $\left\{\bar{\lambda}_{\rho, i}\right\}$ of the ideal closed-loop system and the eigenvalue sensitivity matrices $\left\{\Phi_{\rho, i}\right\}$ were computed. The ASA algorithm was then used to search for an optimal transformation matrix $\mathscr{T}_{\text {opt }}$ by solving for the optimization problem (31) using Theorem 2. This produced the corresponding optimal controller realization $X_{\rho \text {, opt }}$ that maximizes $\mu_{\rho, 1}\left(X_{\rho, \mathscr{T}}\right)$.

Fig. 2 compares the values of $\mu_{\rho, 1}$ given different sampling rates, for the initial and optimal controller realizations. It can be seen from Fig. 2(a) and (b) that optimization effectively improved the FWL closed-loop stability performance for both the $z$ and $\delta$ based controller structures. Fig. 2(c) shows that the optimal $\delta$-based controller realization has a much larger FWL closed-loop stability margin than its $z$-based counterpart. Furthermore, as the sampling rate increases, the stability measure for $X_{\delta \text {, opt improves slightly and eventually }}$ levels out while the stability measure for $X_{z \text {,opt }}$ decreases exponentially. This confirms with a well-known fact that the $\delta$ parameterization has some important advantages over the $z$ parameterization, especially under fast sampling conditions. Fig. 3 depicts the estimated minimum bit lengths for the optimal $z$ and $\delta$ realizations. As mentioned previously, for the $\delta$ parameterization, the sampling period $h$ should be implemented exactly without FWL errors. Even taking into account this requirement, the optimal $\delta$-based realization requires a smaller bit length in FWL implementation. 

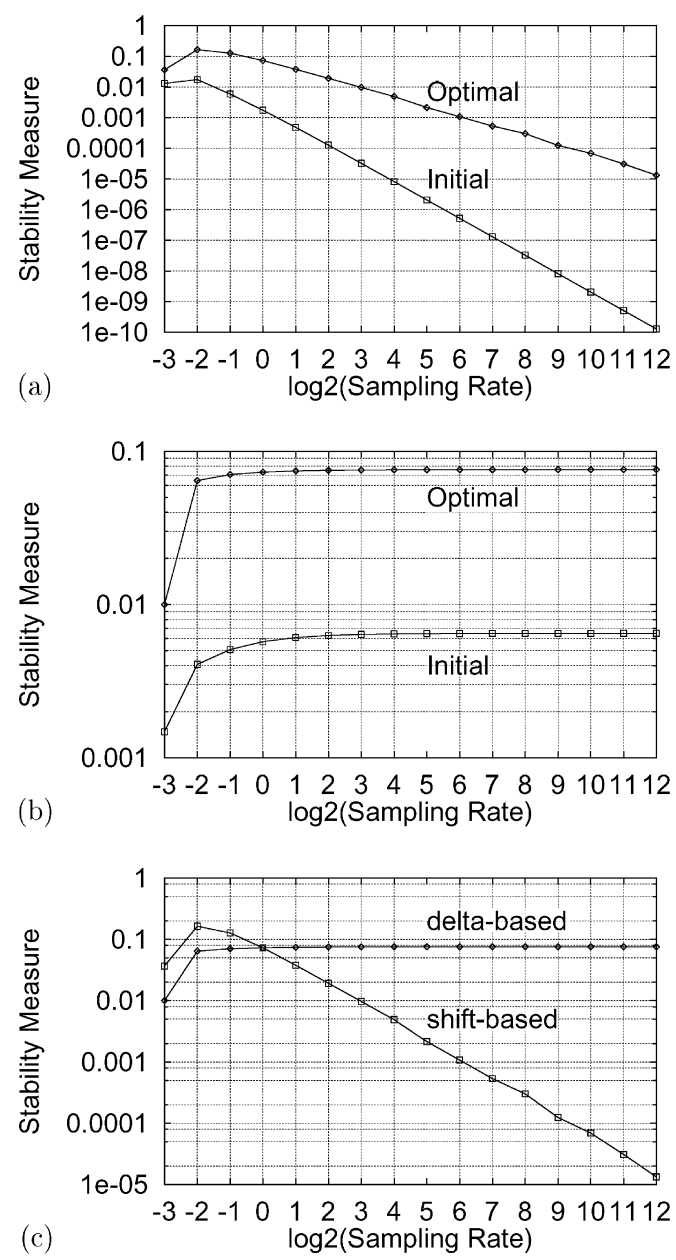

Fig. 2. FWL stability measure $\mu_{\rho, 1}$ as a function of sampling rate for different controller realizations. IFAC93 benchmark PID controller system. (a) Initial and optimal $z$-based controller realizations. (b) Initial and optimal $\delta$-based controller realizations. (c) Optimal $z$ and $\delta$-based controller realizations.

Example 2. This was the control system cited in [5]. The continuous-time plant model was given by

$$
P(s)=\frac{1.6188 s^{2}-0.1575 s-43.9425}{\left(s^{4}+0.1736 s^{3}+27.9001 s^{2}+0.0186 s\right)(s+1)}
$$

and the continuous-time stabilizing controller designed using the $\mathscr{H}_{\infty}$ method was

$$
C(s)=\frac{0.046 s^{6}+1.5862 s^{5}+3.09 s^{4}+44.3 s^{3}+42.7785 s^{2}+0.02867 s+1.58 \times 10^{-4}}{s^{6}+3.766 s^{5}+34.9509 s^{4}+106.2 s^{3}+179.2 s^{2}+166.43 s+0.0033} .
$$

The range of the sampling rate tested in the simulation was $2^{1}$ to $2^{12}$. Again, the discretizing routines in MATLAB were used to obtain the discrete-time plant model $P(\rho)$ and the digital controller $C(\rho)$. The ASA algorithm was used to solve for the resulting optimization problem (27). This produced the corresponding optimal realization $X_{\rho, \text { opt }}$. For this example, the controller order was $n=6$ and the optimization space had a dimension of $n \times n=36$. This was by no means a small task, and the ASA algorithm performed very efficiently.

Fig. 4 compares the values of the FWL stability measure for $X_{z \text {,opt }}$ and $X_{\delta \text {, opt }}$, respectively, under various sampling conditions. Fig. 5 shows the corresponding estimated minimum bit length that can guarantee the 

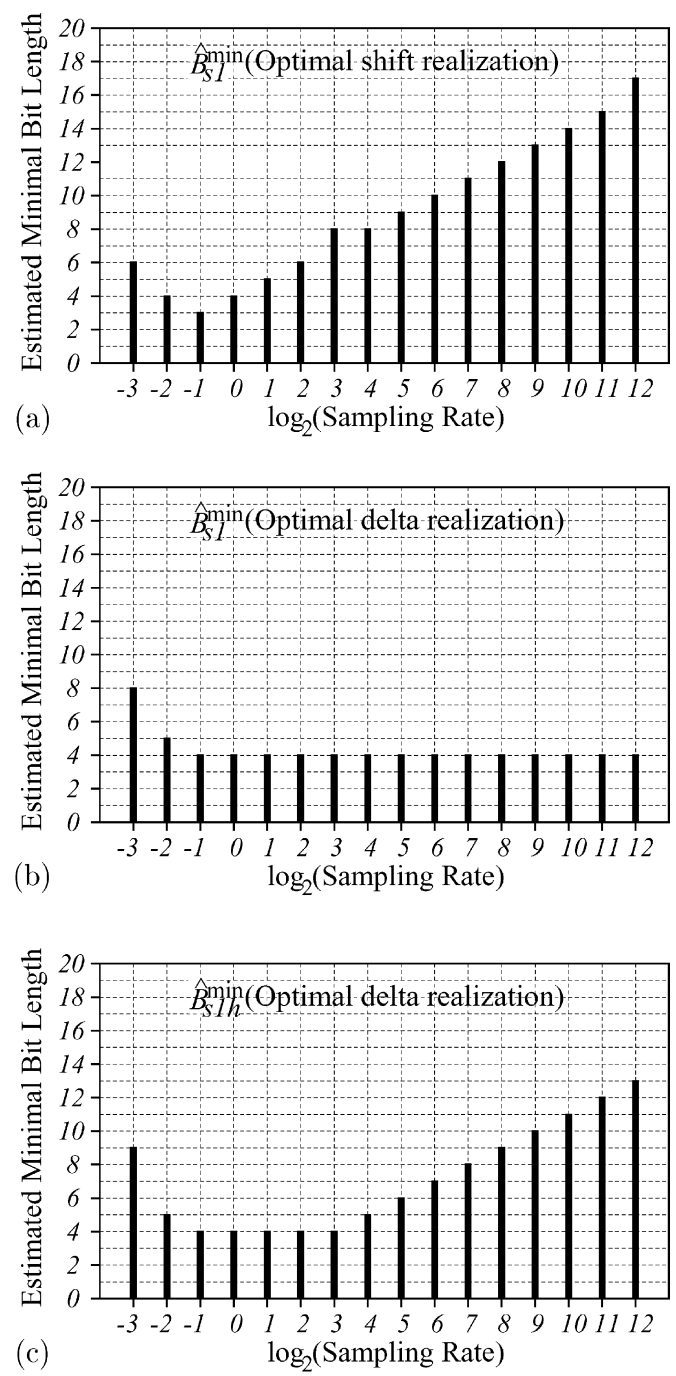

Fig. 3. Estimated minimum bit length as a function of sampling rate for different controller realizations. IFAC93 benchmark PID controller system. (a) $\hat{B}_{\mathrm{S}}^{\mathrm{min}}$ based on $\mu_{z, 1}$ for the optimal $z$-based realization $X_{z \text {,opt }}$. (b) $\hat{B}_{\mathrm{s}}^{\min }$ based on $\mu_{\delta, 1}$ only for the optimal $\delta$-based realization $X_{\delta \text {,opt }}$. (c) $\hat{B}_{\mathrm{s} h}^{\min }$ based on $\mu_{\delta, 1}$ and $h$ for the optimal $\delta$-based realization $X_{\delta \text {,opt }}$.

closed-loop stability for these two controller realizations. In this example, $\hat{B}_{\mathrm{s}}^{\min }=\hat{B}_{\mathrm{s} h}^{\min }$ for the $\delta$ realization $X_{\delta \text {,opt }}$. The results clearly show the advantages of the $\delta$ parameterization over the $z$ operator approach.

\section{Conclusions}

The paper addresses the issues of closed-loop stability of sampled-data control systems with FWL implemented digital controllers. A unified stability measure quantifying the robustness of closed-loop stability to the FWL effects in both the $z$ and $\delta$ domains has been presented. It has been shown that the optimal FWL controller realization can be interpreted as a constrained nonlinear optimization problem. In particular, for PID controller realizations, the optimization can be decoupled into two unconstrained optimization problems. A comparative study using two numerical examples have been given to optimize closed-loop stability bounds 


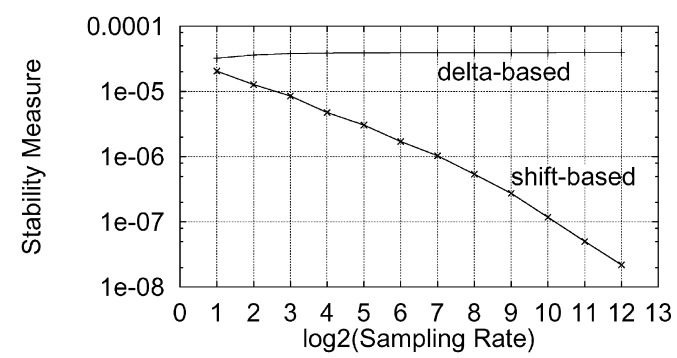

Fig. 4. FWL stability measure $\mu_{\rho, 1}$ as a function of sampling rate for the two different optimal controller realizations. The high-order controller system.
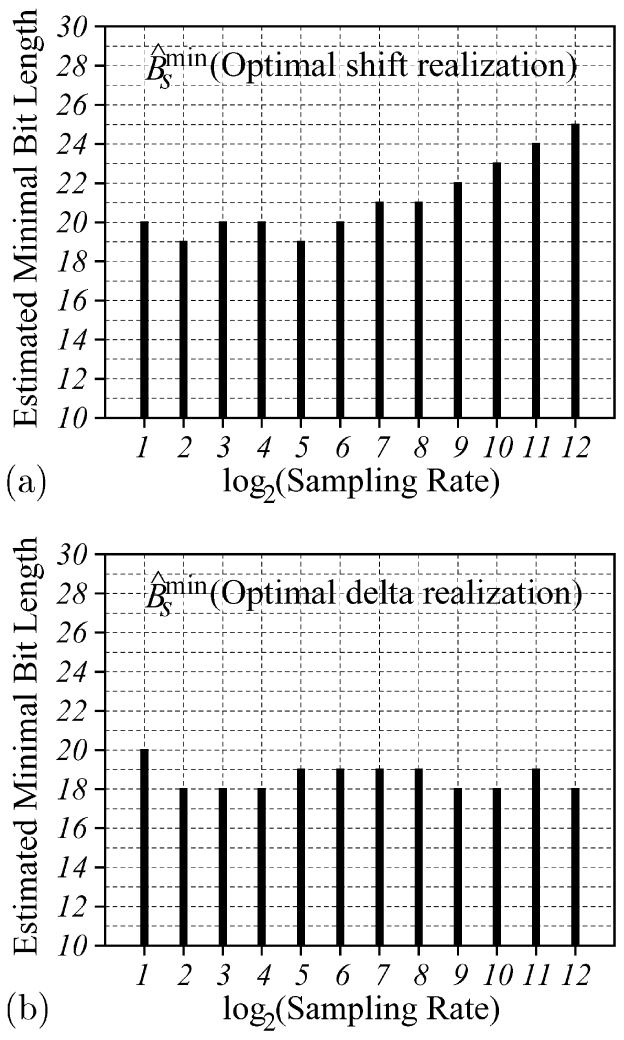

Fig. 5. Estimated minimum bit length as a function of sampling rate for the two different optimal controller realizations. The high-order controller system. (a) $\hat{B}_{\mathrm{s}}^{\mathrm{min}}$ for the optimal $z$-based realization $X_{z \text {,opt }}$. (b) $\hat{B}_{\mathrm{s}}^{\min }\left(=\hat{B}_{\mathrm{s} h}^{\min }\right.$ ) for the optimal $\delta$-based realization $X_{\delta \text {,opt }}$.

of finite-precision controller structures for both the $z$ and $\delta$ operator parameterizations. The results obtained demonstrate that the digital controllers described with the $\delta$ operator has better closed-loop stability robustness to FWL effects, particularly in fast sampling conditions.

\section{Acknowledgements}

S. Chen gratefully acknowledges financial support from the UK EPSRC under grant GR/M16894. 


\section{References}

[1] G.S.G. Beveridge, R.S. Schechter, Optimization Theory and Practice, McGraw-Hill, New York, 1970.

[2] S. Chen, B.L. Luk, Adaptive simulated annealing for optimization in signal processing applications, Signal Processing 79 (1) (1999) 117-128.

[3] S. Chen, J. Wu, R.H. Istepanian, J. Chu, Optimizing stability bounds of finite-precision PID controller structures, IEEE Trans. Automat. Control 44 (11) (1999) 2149-2153.

[4] S. Chen, J. Wu, R.H. Istepanian, J. Chu, J.F. Whidborne, Optimizing stability bounds of finite-precision controller structures for sampled-data systems in the delta operator domain, IEE Proc. Control Theory Applications 146 (6) (1999) 517-526.

[5] T. Chen, B.A. Francis, Input-output stability of sampled-data systems, IEEE Trans. Automat. Control. 36 (1991) 50-58.

[6] L.C.W. Dixon, Nonlinear Optimisation, The English Universities Press, London, 1972.

[7] I.J. Fialho, T.T. Georgiou, On stability and performance of sampled data systems subject to word length constraint, IEEE Trans. Automat. Control. 39 (12) (1994) 2476-2481.

[8] M. Gevers, G. Li, Parameterizations in Control, Estimation and Filtering Problems: Accuracy Aspects, Springer, London, 1993.

[9] L. Ingber, Simulated annealing: practice versus theory, Math. Comput. Modelling 18 (11) (1993) 29-57.

[10] R.H. Istepanian, G. Li, J. Wu, J. Chu, Analysis of sensitivity measures of finite-precision digital controller structures with closed-loop stability bounds, IEE Proc. Control Theory and Applications 145 (5) (1998) 472-478.

[11] G. Li, On the structure of digital controllers with finite word length consideration, IEEE Trans. Automat. Control 43 (5) (1998) 689-693.

[12] R.H. Middleton, G.C. Goodwin, Digital Control and Estimation. A Unified Approach, Prentice-Hall, Englewood Cliffs, NJ, 1990.

[13] J.F. Whidborne, G. Murad, D.W. Gu, I. Postlethwaite, Robust control of an unknown plant - the IFAC93 benchmark, Int. J. Control 61 (3) (1995) 589-640.

[14] J. Wu, R.H. Istepanian, J. Chu, J.F. Whidborne, S. Chen, J. Hu, Stability issues of finite precision controller structures using the delta operator for sampled data systems, in: Proceedings of the 14th IFAC World Congress, Beijing, China, Vol. Q, July 5-9, 1999, pp. $417-422$. 